\title{
Thermal Property Determination of Gases Based on Temperature Oscillating Technique. An Investigation
}

\author{
A. Badarlis ${ }^{1,2}$, V. Kumar ${ }^{2}$, A. Pfau ${ }^{2}$ A. Kalfas ${ }^{1}$ \\ ${ }^{1}$ Aristotle University of Thessaloniki, Thessaloniki, Greece, \\ A. Badarlis: anastasios.badarlis@flowtec.endress.com \\ ${ }^{2}$ Endress+Hauser Flowtec AG, Reinach, Switzerland
}

\begin{abstract}
Thermal conductivity and thermal diffusivity of fluids are a few of thermo-physical properties which characterize a fluid. Different methods have been developed in previous years for the measurement of these two properties, some of them are transient hot wire, $3 \omega$ and temperature oscillation technique. The current investigation is focused on temperature oscillation technique and had the scope of understanding the principle, its limitation and its applicability to an existing gas thermal flow sensor. The investigation consists of 1-D analytical and 3-D numerical model of the existing sensor. The investigation indicated a dependency of the measurement quantities on the thermal properties of the gases and the cause of the discrepancy between the theoretical and the real sensor model. Furthermore, quantities such as the temperature amplitude of the heater can be used for the measurement of thermal conductivity.
\end{abstract}

Key words: Thermal conductivity, thermal diffusivity, thermal flow meter, temperature oscillation technique.

$\begin{array}{ll}\text { Nomenclature } \\ B & \text { Thermal expansion }[1 / \mathrm{K}] \\ N & \text { Kinematic viscosity }\left[\mathrm{m}^{2} / \mathrm{s}\right] \\ \Omega & \text { Angular frequency }[\mathrm{rad} / \mathrm{s}] \\ \Phi & \text { Phase [rad] } \\ c_{p} & \text { Specific heat capacity } \\ F & \text { Frequency }[\mathrm{Hz}] \\ K & \text { Thermal conductivity }[\mathrm{W} / \mathrm{m} K] \\ I_{c h} & \text { Characteristic length }[\mathrm{m}] \\ P & \text { Pressure }\left[\mathrm{N} / \mathrm{m}^{2}\right] \\ \mathrm{Pr} & \text { Prandtl number } \\ Q & \text { Heat flow }[\mathrm{W}] \\ Q & \text { Heat flux }\left[\mathrm{W} / \mathrm{m}^{2}\right] \\ q_{a} & \text { Heat flux amplitude }\left[\mathrm{W} / \mathrm{m}^{2}\right] \\ R & \text { Radial distance }[\mathrm{m}] \\ R a & \text { Rayleigh number } \\ T & \text { Temperature }[\mathrm{K}] \\ T & \text { Time }[\mathrm{s}] \\ T_{\alpha} & \text { Temperature amplitude }[\mathrm{K}] \\ A & \text { Thermal diffusivity }\left[\mathrm{m}^{2} / \mathrm{s}\right]\end{array}$

\section{Introduction}

General

The information regarding thermo-physical properties can be used in a lot of applications, for the verification of the quality to the control of a process or even for the prediction of thermal response of a system in different conditions.
Different techniques have been used for measuring the thermal properties of fluids and solids, such as thermal, acoustic and optical. The most common are the thermal techniques which are classified in two categories, the steady state and the transient [1] - [2].

The scope of the current investigation was to identify the applicability of existing gas thermal flow sensor chip for the measurement of thermal conductivity and thermal diffusivity by implementing a temperature oscillation technique. The investigation can be divided in two parts, the analytical and the numerical. The work started from the analytical part to understand the physics and the parameters which contribute to the response of the sensor. In the second part the 3D numerical model of the sensor in gaseous environment was investigated. The existing sensor chip is fabricated by IST AG and works as thermal flow meter. The scope was to investigate if this chip can have thermal property meter functionality.

Thermal conductivity was primarily defined in the Fourier's law for heat conduction and describes the rate of which the heat is conducted in a substance. Thermal diffusivity eq. 1 is defined as the ratio between the ability of a substance to conduct the heat and the ability to store the thermal energy. 


$$
\alpha=\frac{k}{\rho c_{p}}
$$

The thermal diffusivity has the same dimensions as the kinematic viscosity $v$ [lenght ${ }^{2} /$ time]. Their ratio $v / a$ indicates the relative ease of momentum and energy transport in flow systems. This quantity is dimensionless and is called Prandtl number, it took its name by the German physicist Ludwig Prandtl.

\section{Fluid Thermal Properties Measurement}

The methods for the thermal properties measurement can vary on basis of various principles, time response, accuracy, range of applicability and the thermodynamic state of the substance. All of the methods are based on a heating mechanism and temperature measurement. The heating mechanism can be a resistance heater or a laser source, while temperature meter elements can be thermocouples, RTD, IR thermo-resistors etc.

Some very common methods which are used for the measurement of fluid thermal properties are transient hot wire, transient plate source methods, $3 \omega$ method, temperature oscillation techniques, optical methods and thermal comparator method. One challenging area of application for all methods are the gaseous environments, due to natural convection, selfheating and the big difference between the thermal properties of the sensor and the thermal properties of the gas.

One of the newest method for the calculation of thermal properties is the $3 \omega$ method. First proposed by Cahill and Pohl in 1987 [3], they tried to develop a method of measuring the thermal conductivity of dielectric solids and substrates. Wang $\mathrm{H}$. et al. [4] applied the method using imprinted heaters. Yusibani E. et al. [5] tried to measure the thermal conductivity of hydrogen gas comparing different mathematical models. The conclusion of their investigation was that especially in gases the effect of the heat capacity of wires should be considered in the modeling of the sensor. Only then the extraction of the thermal conductivity is accurate. Similar conclusions found in the work of Gauthier et al. [6] for a range of different gases.

Temperature oscillation technique plays a significant role in the measurement of thermal diffusivity and thermal conductivity. It is easy to implement it either with hot wires or with thinfilms. Measurement principle of the method is based on a heater which is excited by a periodic heating and one or more temperature meter. Measuring the amplitude attenuation, the phase shift and the amplitude the thermal diffusivity, the thermal conductivity can be calculated.

As already mentioned from the possible approaches to achieve the measurement of the thermal properties, one of the most appealing methods is the temperature oscillation techniques. For this reason was selected to be investigated. An advantage of this method is the similarities with the $3 \omega$ in the geometry and the theory, so the $3 \omega$ can be also applied for the same sensor, but it faces also the limitations of the $3 \omega$ method.

Quite a few researchers investigated the temperature oscillation technique, Bhattacharya et. al. [7] assessed the method for the liquids using Petlier elements as heating/cooling elements to control the process and to reduce the amplitude of excitation. They claimed that they achieved accuracy of $2 \%$, which is one of the most accurate measurements based on this method. An older investigation of this method in liquids was the work of Czarnetzki et. al. [8]. Their paper presents the analytical solution of the heat equation for simplified geometries and different boundary conditions, they managed to achieve accuracy of $2 \%$ for the thermal diffusivity and $8 \%$ for the thermal conductivity. A big number of publications, related to this method, are dedicated to applications of nanofluids such as Patel et al. [9]. Temperature oscillation technique in gases has been conducted by Reyes-Romero et al. [10], achieving to determine the thermal conductivity of a flowing gas and simultaneously its velocity for flow speeds up to $1 \mathrm{~m} / \mathrm{s}$. Beigelbeck R. et al. [11] in their work managed to measure both of the properties for a laminar flowing gas. They presented a very detail analytical model for their sensor and fitting the experimental data to the analytical model they achieve to measure the thermal conductivity and diffusivity.

\section{Methods and Materials}

\section{Analytical Model}

Having in mind of an analytical solution, which is closer to the chip sensor, the 1-D problem of heat transfer in spherical coordinated was adopted [12]. Only heat conduction is considered and the domain has homogenous and continuous properties. The solution of the heat equation (eq. 4) describes the temperature distribution in the domain. The domain has the characteristics of the semi-infinite body, while the radius of the source is $R$. The heat which is generated in the source has the form of eq. 2 . 


$$
\begin{gathered}
Q=Q_{0} \cos (\omega t) \\
T=T_{r}-T_{\infty} \\
\frac{\partial^{2} T}{\partial r^{2}}+\frac{2}{r} \frac{\partial T}{\partial r}-\frac{1}{\alpha} \frac{\partial T}{\partial t}=0
\end{gathered}
$$

The boundary conditions of the problem are the eq. 5 and eq. 6

$$
\begin{gathered}
Q_{0} \cos (\omega t)=-\left.k 2 \pi R^{2} \frac{\partial T}{\partial r}\right|_{r=R} \\
T(\infty, t)=0
\end{gathered}
$$

As the problem is periodic steady state, substituting the periodic solution, the PDE turns to ODE (eq.7)

$$
\frac{\mathrm{d} T_{a}}{\mathrm{~d} r^{2}}+\frac{2}{r} \frac{\mathrm{d} T_{a}}{\mathrm{~d} r}-\frac{i \omega}{\alpha} T_{a}=0
$$

Where $T_{\alpha}(r)$ is the amplitude of the temperature in the domain [12]. Solving this ODE and combining with the solution in the time domain two cases can be extracted. The first case is the special case where the $R \rightarrow 0$, eq. 8 and the general case (eq. 9) for finite radius (R) of the source.

$$
\begin{gathered}
T=\frac{Q_{0}}{4 \pi k r} e^{-\sqrt{\frac{\omega}{2 \alpha}} r} \cos \left(\omega t-\sqrt{\frac{\omega}{2 \alpha}} r\right) \\
T=\frac{Q_{0}}{4 \pi k r} \sqrt{\left(R \sqrt{\frac{\omega}{2 \alpha}}+1\right)^{2}+\left(R \sqrt{\frac{\omega}{2 \alpha}}\right)^{2}} \\
e^{-\sqrt{\frac{\omega}{2 \alpha}}(r-R)} \cos \left(\omega t-\sqrt{\frac{\omega}{2 \alpha}}(r-R)+\phi\right) \\
\phi=\arctan \left(\frac{-1}{\left.1+\frac{1}{R \sqrt{\frac{\omega}{2 \alpha}}}\right)}\right)
\end{gathered}
$$

From the solution of the 1-D problem we can conclude that the phase is dependent only on the thermal diffusivity, while the amplitude contains both of the properties. We can also infer that the dependency of amplitude on thermal diffusivity reduces if the following conditions are satisfied, first the measurement point of interest is close to the source $r \rightarrow R$ and second if $R \sqrt{\omega /(2 \alpha)}<1$.

$\sqrt{\omega / \alpha}$ characterizes the system and has the name of thermal diffusion length or thermal penetration depth. It is considered as an estimation of the distance, at which an appreciable amount of energy is transferred.

\section{Numerical Model}

The 3-D model consists of the sensor chip Fig. 1 and the fluid domain where the chip is placed. After a grid investigation the number of 200000 volume elements was the optimum.
The sensor chip consists of one heater and two temperature meters, one is placed in short distance from the heater $(0.465 \mathrm{~mm})$, named as "Tshort" and the other in long distance $(0.695$ $\mathrm{mm}$ ), named as "Tlong", all made of platinum. The elements are protected from corrosion with two polyimide layers on both sides of the sensor. The thickness of each layer is $5 \mu \mathrm{m}$ and its properties are $\mathrm{k}: 0.12 \mathrm{~W} / \mathrm{mK}, c_{p}: 1090 \mathrm{~J} / \mathrm{kgK}$ and $\rho: 1430 \mathrm{~kg} / \mathrm{m}^{3}$.

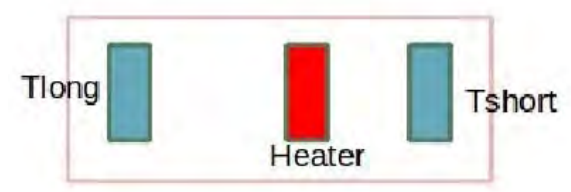

Fig. 1. Sensor chip. One heater in the middle and two temperature meter on both sides.

As the temperature oscillation technique is dynamic effect, transient type of solution of conjugate heat transfer problem was applied. The excitation on the heater had the form $q$ $=500(\sin (\omega t)+1)\left[\mathrm{W} / \mathrm{m}^{2}\right]$ and frequency of $1 \mathrm{~Hz}$. The model was simulated for 6 periods and the simulation time step was $0.02 \mathrm{sec}$ to achieve a good discretization.

Although the natural convection was considered into the model, its impact on the results is very low and negligible. This happens because the excitation power selected to be low avoiding other effect such as natural convection or properties variation due to temperature variation.

The data of the numerical simulation was analyzed with the help of scipy a package of python programming language. Two different approaches were applied on the output of the simulation data. These approaches correspond to different transform, the fast Fourier transform and the Hilbert transform. In this paper, results only from FFT are presented.

\section{Results and Discussion}

\section{Analytical Model}

Firstly, to understand the impact of the parameters on the response of the system, graphs of the analytical solution (eq.9) were drawn.

In Fig. 2 (excitation of $1 \mathrm{~Hz}$ ) and Fig. 3 (excitation of $200 \mathrm{~Hz}$ ) amplitude and phase are plotted against the thermal conductivity. We can identify the impact of frequency on amplitude and phase. The graphs present 4 cases of different densities $(0.1,1,100,1000)$ or in other words different diffusivities, as it is related to density by eq.1. It implies that in high frequencies the impact of the diffusivity on the 
amplitude is higher. On the diagrams the lines of amplitude are merging earlier for $1 \mathrm{~Hz}$ rather than $200 \mathrm{~Hz}$. This means that lower frequency excitation is more desirable since the amplitude is dependent only on conductivity. On the other hand in low excitation frequencies the penetration depth is longer. Additionally, the phase sensitivity is higher in higher frequencies.

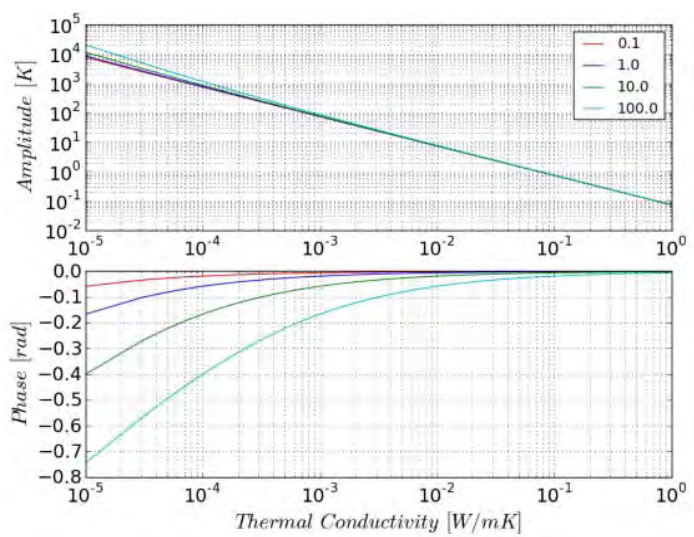

Fig. 2. Amplitude and phase against thermal conductivity for four different densities. Excitation frequency of $1 \mathrm{~Hz}$.

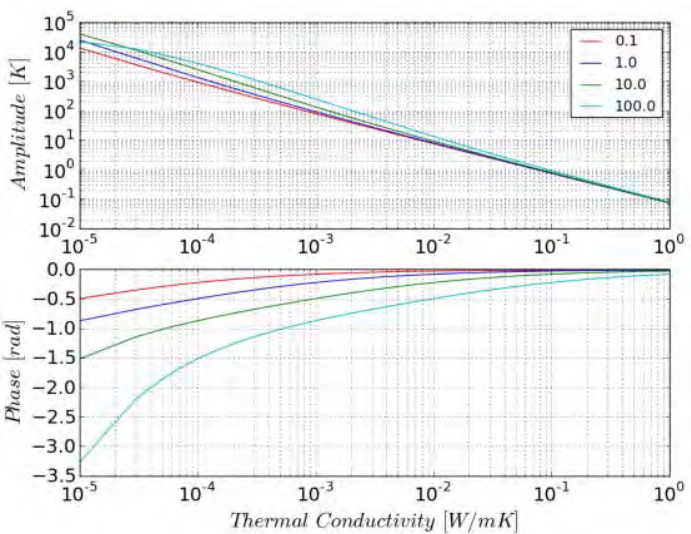

Fig. 3. Amplitude and phase against thermal conductivity for four different densities. Excitation frequency of $200 \mathrm{~Hz}$.

\section{Numerical Model}

For the numerical investigation two groups of gases were used. The first group had mixtures with the same thermal conductivity, while at the second group the thermal diffusivity was the same. Each group contains 7 different mixtures. More data about the mixtures composition and properties can be found in the table 1 and 2 . Fig. 4 presents the temperature distribution of a section in the fluid domain and on the chip surface. The form of the isothermals is spherical which resembles the spherical distribution of 1-D analytical model.

In the following figures the spectrum analysis of heater and temperature meter in long distance "Tlong" are presented.

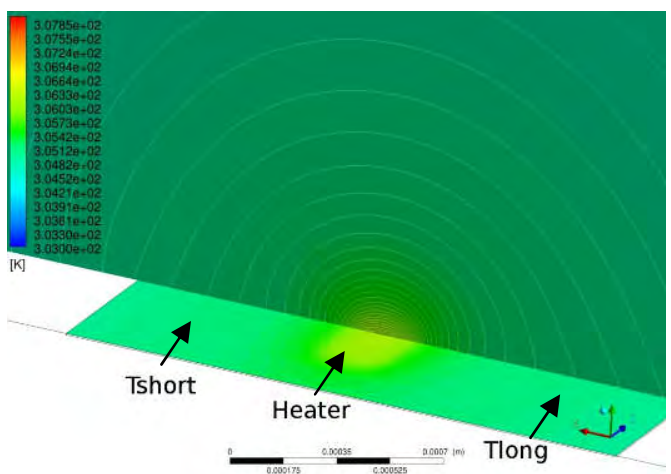

Fig. 4. Temperature distribution in fluid domain and on the surface of the chip.

Tab. 1: Group of gases with the same thermal conductivity

\begin{tabular}{|l|c|c|c|c|c|c|c|}
\hline & $\mathrm{Xe}-\mathrm{He}$ & $\mathrm{CO} 2-\mathrm{CH} 4$ & Air-CO2 & $\mathrm{Ar}-\mathrm{H} 2$ & $\mathrm{Ne}-\mathrm{NH} 3$ & $\mathrm{HCl}-\mathrm{CH} 4$ & $\mathrm{H} 2 \mathrm{~S}-\mathrm{N} 2$ \\
\hline$\%$ & $65-35$ & $45-55$ & $99-1$ & $91-9$ & $5.5-95.4$ & $52-47$ & $20-80$ \\
\hline$\rho\left(\mathrm{kg} / \mathrm{m}^{3}\right)$ & $3.5 \mathrm{E}+00$ & $1.2 \mathrm{E}+00$ & $1.2 \mathrm{E}+00$ & $1.5 \mathrm{E}+00$ & $6.9 \mathrm{E}-01$ & $1.1 \mathrm{E}+00$ & $1.1 \mathrm{E}+00$ \\
\hline $\mathrm{C}_{\mathrm{p}}(\mathrm{J} / \mathrm{kg} \cdot \mathrm{K})$ & $2.4 \mathrm{E}+02$ & $1.3 \mathrm{E}+03$ & $1.0 \mathrm{E}+03$ & $5.9 \mathrm{E}+02$ & $2.1 \mathrm{E}+03$ & $1.2 \mathrm{E}+03$ & $1.0 \mathrm{E}+03$ \\
$\kappa(\mathrm{W} / \mathrm{m} . \mathrm{K})$ & $2.6 \mathrm{E}-02$ & $2.6 \mathrm{E}-02$ & $2.6 \mathrm{E}-02$ & $2.6 \mathrm{E}-02$ & $2.6 \mathrm{E}-02$ & $2.6 \mathrm{E}-02$ & $2.6 \mathrm{E}-02$ \\
\hline$\mu\left(\mathrm{N} \mathrm{s} / \mathrm{m}^{2}\right)$ & $2.5 \mathrm{E}-05$ & $1.3 \mathrm{E}-05$ & $1.9 \mathrm{E}-05$ & $2.3 \mathrm{E}-05$ & $1.1 \mathrm{E}-05$ & $1.3 \mathrm{E}-05$ & $1.8 \mathrm{E}-05$ \\
$\alpha\left(\mathrm{m}^{2} / \mathrm{s}\right)$ & $3.1 \mathrm{E}-05$ & $1.8 \mathrm{E}-05$ & $2.2 \mathrm{E}-05$ & $3.0 \mathrm{E}-05$ & $1.8 \mathrm{E}-05$ & $2.0 \mathrm{E}-05$ & $2.3 \mathrm{E}-05$ \\
\hline
\end{tabular}
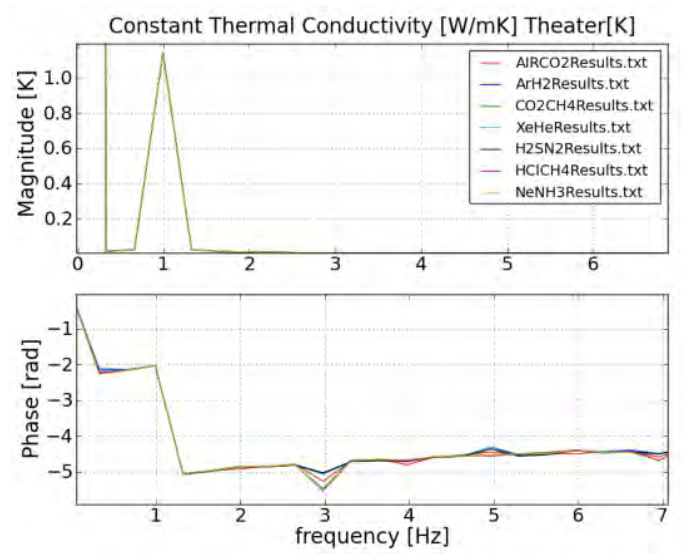

Fig. 5. Spectrum analysis for the temperature response of the heater for the mixtures with the same thermal conductivity Maximum deviation from the mean value, magnitude $\pm 0.43 \%$, phase $\pm 0.5 \%$.
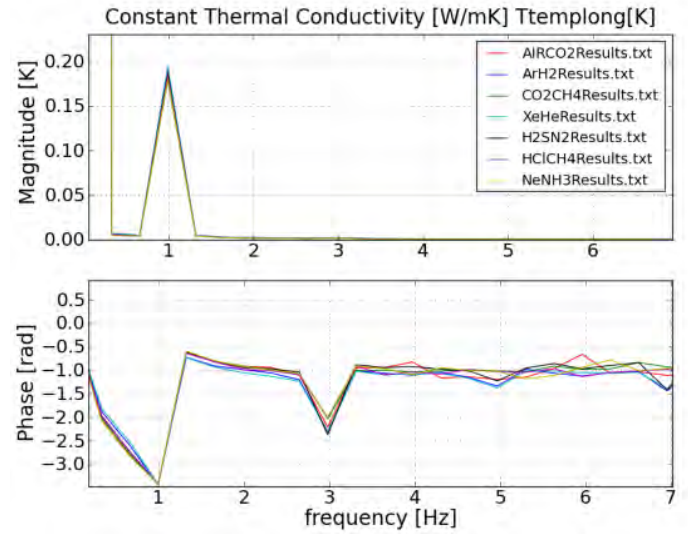

Fig. 6. Spectrum analysis for the temperature response of the temperature meter in long distance "Tlong" for the mixtures with the same thermal conductivity. Maximum deviation from the mean value, magnitude $\pm 8 \%$, phase $\pm 0.2 \%$. 
For the group of mixtures with the same thermal conductivity, the amplitude of the heater Fig. 5 and the phase of temperature meter Fig. 6 have the lowest sensitivity to thermal diffusivity.

Comparing the magnitude on the heater and temperature meter (Fig. 5 and Fig. 6), the impact of the distance from the source can be identified, since the response of the heater is influenced less by diffusivity variation than the response of the temperature meter. This is in agreement with the results of 1-D analytical model for the case where $r \rightarrow R$.

Tab. 2: Group of gases with the same thermal diffusivity.

\begin{tabular}{|c|c|c|c|c|c|c|c|}
\hline & Xe-He & CO2-CH4 & Air-CO2 & $\mathrm{Ar}-\mathrm{H} 2$ & Ne-NH3 & $\mathrm{HCl}-\mathrm{CH} 4$ & H2S-N2 \\
\hline$\%$ & $75-25$ & $13-87$ & $99-1$ & $99-1$ & $20-80$ & $28-72$ & $27-73$ \\
\hline$\rho\left(\mathrm{kg} / \mathrm{m}^{3}\right)$ & $4.0 \mathrm{E}+00$ & $7.9 \mathrm{E}-01$ & $1.2 \mathrm{E}+00$ & $1.6 \mathrm{E}+00$ & $7.1 \mathrm{E}-01$ & $8.8 \mathrm{E}-01$ & $1.1 \mathrm{E}+00$ \\
\hline$c_{p}(J / k g . K)$ & $2.1 \mathrm{E}+02$ & $1.8 \mathrm{E}+03$ & $1.0 \mathrm{E}+03$ & $5.3 \mathrm{E}+02$ & $1.9 \mathrm{E}+03$ & $1.6 \mathrm{E}+03$ & $1.0 \mathrm{E}+03$ \\
\hline$\kappa(\mathrm{W} / \mathrm{m} . \mathrm{K})$ & $1.9 \mathrm{E}-02$ & $3.2 \mathrm{E}-02$ & $2.6 \mathrm{E}-02$ & 1.9E-02 & $3.0 \mathrm{E}-02$ & 3.0E-02 & $2.6 \mathrm{E}-02$ \\
\hline$\mu\left(\mathrm{N} \mathrm{s} / \mathrm{m}^{2}\right)$ & $2.5 \mathrm{E}-05$ & $1.2 \mathrm{E}-05$ & $1.9 \mathrm{E}-05$ & 2.3E-05 & $1.4 \mathrm{E}-05$ & $1.3 \mathrm{E}-05$ & $1.7 \mathrm{E}-05$ \\
\hline$\alpha\left(\mathrm{m}^{2} / \mathrm{s}\right)$ & 2.2E-05 & 2.2E-05 & $2.2 \mathrm{E}-05$ & 2.2E-05 & $2.2 \mathrm{E}-05$ & 2.2E-05 & $2.2 \mathrm{E}-05$ \\
\hline
\end{tabular}
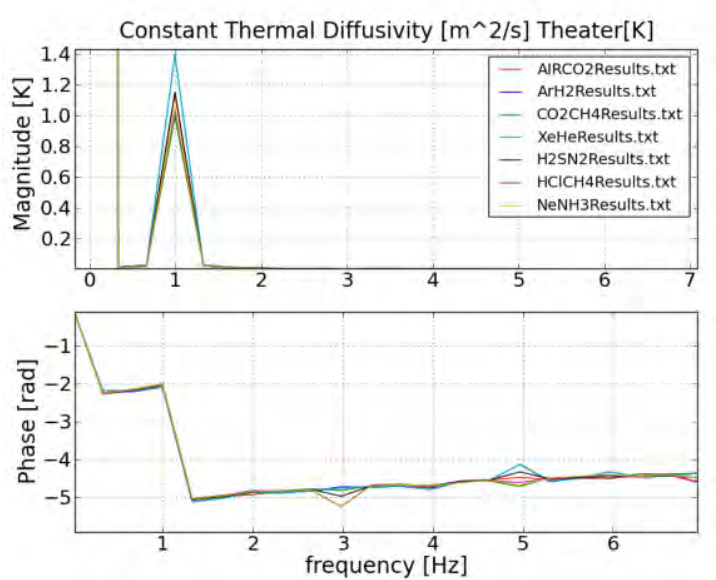

Fig. 7. Spectrum analysis for the temperature response of the heater for the mixtures with the same thermal diffusivity. Maximum deviation from the mean value magnitude $\pm 40 \%$, phase $\pm 4 \%$.
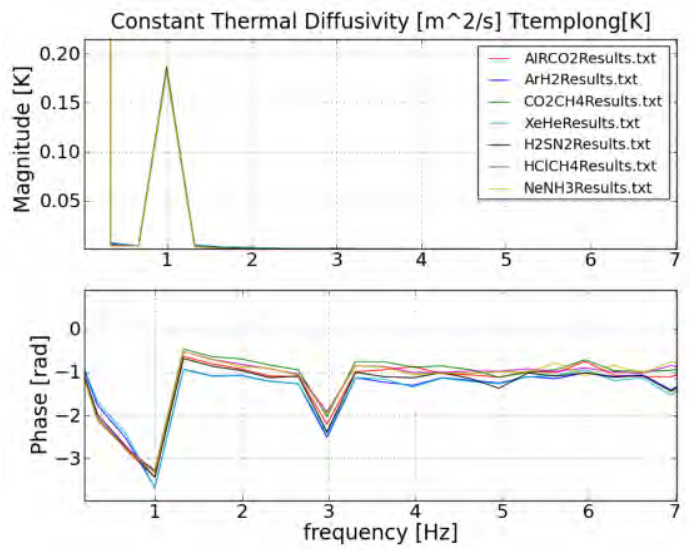

Fig. 8. Spectrum analysis for the temperature response of the temperature meter in long distance "Tlong" for the mixtures with the same thermal diffusivity. Maximum deviation from the mean value magnitude $\pm 2.7 \%$, phase $\pm 12 \%$.
In the group of mixtures with common value of thermal diffusivity Fig. 7 and Fig. 8 none from the amplitude or phase is independent of thermal conductivity. As all of them vary, the maximum deviation from the mean value is high. This means that the phase for the sensor model is dependent on thermal conductivity and diffusivity, this comes in contrast to homogenous 1-D model of eq. 9, where the phase contains only the thermal diffusivity.

\section{Numerical Model without Polyimide Layer}

To understand the discrepancy between the numerical and the analytical model the polyimide layer was removed from the model. This means that the elements are direct in contact with the fluid domain and there is no discontinuity or inhomogeneity in the computation domain. The platinum elements are modeled as interfaces. The results of this simulation for 4 mixtures with the same thermal diffusivity are presented in Fig. 9. The trend of the results for this investigation agrees with the trend of the 1-D analytical model.
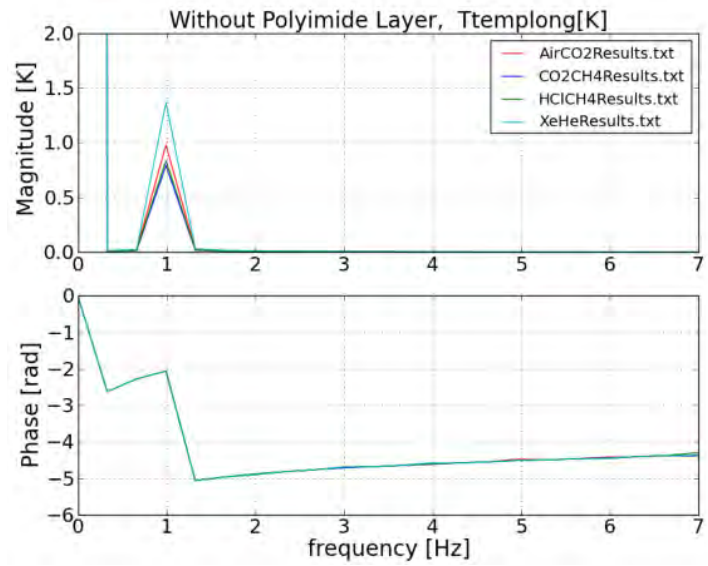

Fig. 9. Spectrum analysis of the temperature response of the temperature meter in long distance "long" for the mixtures with the same thermal diffusivity. Phase exhibits the same response for all the mixtures, agreement with the1-D homogenous model theory.

According to the results polyimide layer has a decisive impact on the response of the system. Polyimide protection layer introduces discontinuity in the system and increases the thermal inertia. Furthermore polyimide layer reduces the amplitude of the signal.

\section{Conclusions}

Summarizing the most important points of this investigation:

- The thermal conductivity can be determined directly from the amplitude of heater temperature or the phase of the temperature meter since these two 
quantities are the less sensitive to thermal diffusivity variations.

- Discrepancy between the 1-D analytical and the numerical model of the sensor chip, due to variation of system thermal inertia and the introduction of inhomogeneity and discontinuity.

- Polyimide has very low thermal conductivity which lowers the measurement signal. To increase the sensitivity of the sensor materials with higher thermal conductivity or thinner layer can be used.

- To improve the accuracy of the determination of thermal conductivity and thermal diffusivity, a model which describes accurately the behavior of the sensor should be developed, since none from amplitude and phase seems to be dependent only in one property.

- The sensor design can be optimized with criteria of higher functional range of frequency and modeling, because simple design is modeled easier.

\section{References}

[1] K. Goodson and M. Flik, Solid layer thermal conductivity measurement techniques, ASME, Appl Mech Rev, vol. 47, no 3, (1994).

[2] C. Kleinstreuer and Y. Feng, Experimental and theoretical studies of nano-fluid thermal conductivity enhancement: a review, Nanoscale Research Letters, SpingerOpen, pp. 19-30 (2011).

[3] R. Pohl and D. G. Cahill, Thermal conductivity of amorphous solids, Applied and Environmental Microbiology, vol. 69, pp. 4067-4073 (2003).

[4] H. Wang and M. Sen, Analysis of the 3-omega method for thermal conductivity measurement, Int. J. of Heat Transfer, vol. 52, pp. 2102-2109, (2009).

[5] E. Yusibani, P.L. Woodfield, M. Fujii, K. Shinzato, x. Zhang, Y. Takata, Application of the threeomega method to measurement of thermal conductivity and thermal diffusivity of hydrogen gas, Int j Thermophys, 30, 397-415, (2009).

[6] S. Gauthier, A. Giani and P. Combette, Gas hermal conductivity measurement using a $3 \omega$ based sensor, Design, Test, Integration and Packaging of MEMS/MOEMS (DTIP), IEEE, 160163 (2012).

[7] P. Bhattacharya, S. Nara, P. Vijayan, T. Tang, W. Lai, R. Prasher, D. Song, and J. Wang, Characterization of the temperature oscillation technique to measure the thermal conductivity of fluids, Int. Journal of Heat and Mass Transfer, vol. 49, pp. 2950-2956 (2008).
[8] W. Czarnetzki and W. Roetzel, Temperature oscillation techniques for simultaneous measurement of thermal diffusivity and conductivity, Int. Journal of Thermophysics, vol. 16 No 2 (1995).

[9] H. Patel, T. Sundararajan, and S. Das, An experimental investigation into the thermal conductivity enhancement in oxide and metallic nano-fluids, Journal of Nanoparticle Research, vol. 12, pp. 1015-1031 (2010).

[10] D.F. Reyes-Romero, A.S. Cubukcu and G.A. Urban, Simulation of the oscillatory excitation of a thermal flow sensor, $1^{\text {st }}$ Int. Conference on Microfluidic Handling Systems, 22-25 (2011).

[11] R. Beigelbeck, F. Kohl, S. Cerimovic, A. Talic, F. Keplinger and B. Jakoby, Thermal property determination of laminar-flowing fluids utilizing the frequency response of a calorimetric flow sensor, IEEE Sensors 2008, Lecee, Italy, 518521 (2008).

[12] D. H. Baehr and K. Stephan, Wärme- und Stoffübertragung. Berlin Heidelberg: Springer, 4th ed., (2004).

\section{Acknowledgement}

The authors would like to thank Endress+Hauser Flowtec AG for the permission of publishing this work. 